\title{
Characterization of slanted buried planar heat sources using time domain Infrared Thermography
}

\author{
by A. Mendioroz*, A. Salazar*, K. Martinez**, A. Cifuentes**, E. Marín**, R. Celorrio***, P. \\ Venegas $^{\star \star \star *}$ I. Sáez de Ocáriz ${ }^{\star \star \star *}$
}

* Departamento de Física Aplicada I, Escuela Técnica Superior de Ingeniería, Universidad del País Vasco UPV/EHU, Pza. Ingeniero Torres Quevedo 1, 48013 Bilbao, Spain E-mail: arantza.mendioroz@ehu.eus

**Instituto Politécnico Nacional, (IPN), Centro de Investigación en Ciencia Avanzada y Tecnología Avanzada (CICATA), Unidad Legaria, Legaria 694, Col. Irrigación, C. P. 11500, Ciudad de México, Mexico.

***Departamento de Matemática Aplicada, EINA/IUMA, Universidad de Zaragoza, Campus Río Ebro, Edificio Torres Quevedo, 50018 Zaragoza, Spain.

**** Centro de Tecnologías Aeronáuticas CTA Parque Tecnológico de Alava, Miñano, Spain

\begin{abstract}
We address the characterization of planar inner defects of any inclination, using infrared thermography (IRT) with any kind of excitation that turns the defect into a heat source (ultrasonic or eddy current). First, we calculate the surface temperature distribution. Then, we present multi-parametric fittings (dimensions, depth and inclination) of noisy synthetic data to characterize the defect. Finally, we prepare 3D printed plastic parallelepiped samples containing graphite and copper slabs that are excited optically and inductively, respectively. The fittings of the data to the model confirm the ability of IRT to characterize planar defects with any inclination.
\end{abstract}

\section{Introduction}

In recent years, infrared thermography with ultrasonic excitation has proven to be a very useful tool to characterize the dimensions and depth of vertical cracks, both in lock-in [1] and burst regimes [2]. In this technique, when the sample is excited with ultrasounds the defect behaves as a heat source, due to the local conversion of mechanical into thermal energy. In this work, we present a first approximation to extend previous works (in time domain) with the aim of characterizing any type of planar defect of arbitrary inclination, using infrared thermography with any excitation that makes the defect behave as a heat source. Some examples could be inclined kissing cracks excited with ultrasounds or planar metallic inclusions within an insulator excited inductively. The characterization is tackled as a parameter identification problem, assuming knowledge of the heat source geometry.

As a first step, we calculate the evolution of the surface temperature distribution produced by step-heated-slanted buried planar heat sources of different geometries and we present simulations to illustrate the effect of the dimensions, depth and inclination on the surface temperature distribution. Then, in order to test the ability of the method to characterize planar slanted heat sources, we generate noisy synthetic data and we fit them to estimate the lateral dimensions, the depth and the angle of inclination of the heat source. Finally, we prepare 3D printed plastic (optically transparent) parallelepiped samples, containing embedded $\mathrm{Cu}$ or graphite slabs of different shapes and inclinations. We generate calibrated inner heat sources with two kinds of excitations: the graphite slabs are excited with a laser beam and the Cu slabs with an induction system. The results of the fittings of the data to the model confirm the ability of IRT to characterize inner heat sources that represent defects of any inclination and shape.

\section{Theory}

We-calculated the evolution of the surface temperature distribution produced by a rectangular heat source of width $w$ and height $h$ contained in plane $\Pi$ that makes an angle $\varphi$ with the sample surface. The upper side of the rectangle is buried at a depth $d$. The rectangle emits a constant and homogeneous heat flux of intensity lo during a time interval $\tau$ and the sample is assumed as semi-infinite. The geometry is shown in figure 1.
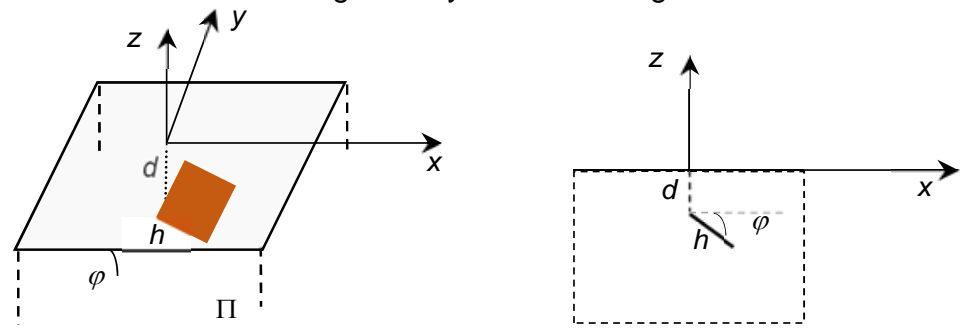

Fig. 1: Geometry of a rectangular heat source of width $w$ and height $h$ contained in plane $\Pi$ that makes an angle $\varphi$ with the surface. The upper side of the rectangle is buried at a depth $d$ below the surface. 
The temperature at the surface of a material of thermal conductivity, $K$, and diffusivity, $D$, can be easily obtained by generalizing the calculation corresponding to a vertical heat source [2]:

$$
\begin{gathered}
T_{1}\left(\vec{r}_{z=0}, t\right)=\int_{-w / 2}^{w / 2} \int_{0}^{h \cos \varphi} \frac{I_{o}}{2 \pi K} \frac{\sqrt{1+m^{2}}}{\sqrt{\left(x-x^{\prime}\right)^{2}+\left(y-y^{\prime}\right)^{2}+\left(z-\left(m x^{\prime}-d\right)\right)^{2}}} \operatorname{Erfc} \frac{\sqrt{\left(x-x^{\prime}\right)^{2}+\left(y-y^{\prime}\right)^{2}+\left(z-\left(m x^{\prime}-d\right)\right)^{2}}}{\sqrt{4 D t}} d x^{\prime} d y^{\prime} \quad 0 \leq t \leq \tau \\
T_{2}\left(\vec{r}_{z=0}, t\right)=\int_{-w / 2}^{w / 2} \int_{0}^{h \cos \varphi} \frac{I_{o}}{2 \pi K} \frac{\sqrt{1+m^{2}}}{\sqrt{\left(x-x^{\prime}\right)^{2}+\left(y-y^{\prime}\right)^{2}+\left(z-\left(m x^{\prime}-d\right)\right)^{2}}} \times \\
{\left[\operatorname{Erfc} \frac{\sqrt{\left(x-x^{\prime}\right)^{2}+\left(y-y^{\prime}\right)^{2}+\left(z-\left(m x^{\prime}-d\right)\right)^{2}}}{\sqrt{4 D t}}-\operatorname{Erfc} \frac{\sqrt{\left(x-x^{\prime}\right)^{2}+\left(y-y^{\prime}\right)^{2}+\left(z-\left(m x^{\prime}-d\right)\right)^{2}}}{\sqrt{4 D(t-\tau)}}\right] d x^{\prime} d y \quad 0 \leq t \leq \tau}
\end{gathered}
$$

where $m=\tan \varphi$.

\section{Experiments and fittings of experimental data}

In order to check the ability of the method to characterize inclined cracks we have prepared 3D printed photopolymer resin (PR48) samples (optically transparent, $D=0.13 \mathrm{~mm}^{2} / \mathrm{s}, K=0.5 \mathrm{~W} / \mathrm{mK}$ ) with embedded copper and graphite slabs of triangular and rectangular shape and different inclinations with respect to the surface, namely $0^{\circ}, 30^{\circ}$, $45^{\circ}, 60^{\circ}$, and $90^{\circ}$. The samples containing graphite slabs were excited by illuminating the slab laterally with a diode laser emitting at $530 \mathrm{~nm}$. The samples containing copper slabs were placed in the middle of an $8 \mathrm{~cm}$ coil fed with currents ranging from 5 to $15 \mathrm{~A}$ at $120 \mathrm{kHz}$. Measurements have been performed using FLIR SC 5000 thermographic camera. In figure 2 we present experimental data and fittings to the model corresponding to a copper slab of width $w=2.1 \mathrm{~mm}$ and height $h=2 \mathrm{~mm}$, buried at a depth $d=0.8 \mathrm{~mm}$, that is inclined $\varphi=45^{\circ}$ with respect to the sample surface. Data correspond to $\mathrm{a} \tau=4 \mathrm{~s}$ burst. The OX and OY profiles captured at the end of the burst (Fig. 2a) and the temperature evolution at point $(0,0,0)$ (Fig. 2b) are fitted to Eqs. 1 with $I_{0} / K, w, h, d$, and $\varphi$ as fitting parameters. The agreement between the fitted and actual geometrical parameters defining the cracks opens the possibility of using IRT to characterize planar inner heat sources representative of inclined defects excited in IRT experiments.
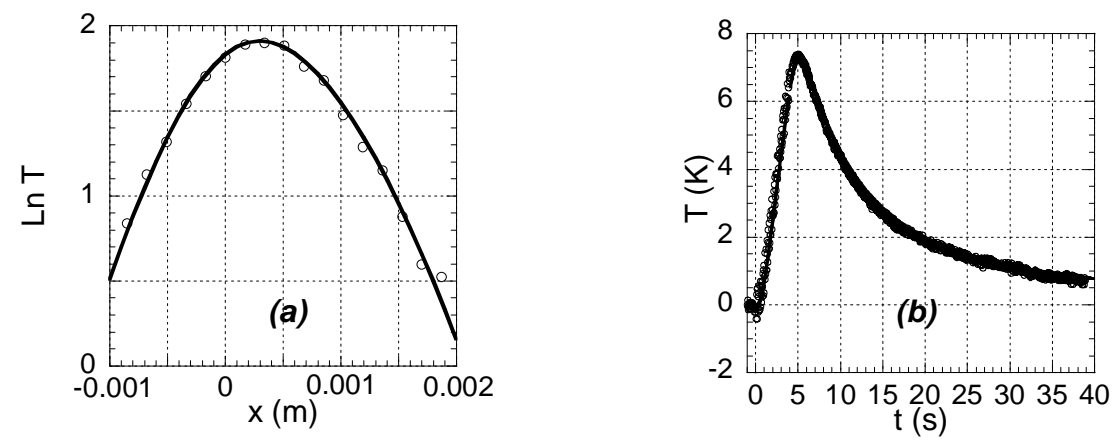

Fig. 2: Experimental (symbols) OX temperature profile obtained at the end of a $\tau=4 \mathrm{~s}$ burst (a) and temperature evolution at $(0,0,0)(b)$ corresponding to a sample that contains a copper slab of width $\mathrm{w}=2.1 \mathrm{~mm}$, height $h=2 \mathrm{~mm}$, that is buried $d=0.8 \mathrm{~mm}$ and inclined $\varphi=45^{\circ}$ with respect to the surface. The solid line is the fitting to Eqs.1

The authors would like to acknowledge financial support by Ministerio de Economía y Competitividad (DPI2016-77719-R, AEI/FEDER, UE), by Universidad del País Vasco UPV/EHU (GIU16/33), SIP-IPN (Research Grants (20160144, 20170545) and CONACyT (2015-02-1042, 264093, 2016-01-2482). The support of COFAA-IPN by the SIBE and BEIFI programs is also acknowledged.

\section{REFERENCES}

[1] Castelo A., Mendioroz A., Celorrio R., Salazar A. Optimizing the inversion protocol to Determine the geometry of vertical cracks from lock-in vibrothermography. J Nondestruct Eval 2017;36:3 (12 pp).

[2] Mendioroz A.,Celorrio R., Cifuentes A., Zatón L., Salazar A. Sizing vertical cracks using burst vibrothermography. NDT\&E Int. 2016;84:36-46. 